\title{
BASES AND BOREL SELECTORS FOR TALL FAMILIES
}

\author{
JAN GREBÍK AND CARLOS UZCÁTEGUI
}

\begin{abstract}
Given a family $\mathcal{C}$ of infinite subsets of $\mathbb{N}$, we study when there is a Borel function $S: 2^{\mathbb{N}} \rightarrow 2^{\mathbb{N}}$ such that for every infinite $x \in 2^{\mathbb{N}}, S(x) \in \mathcal{C}$ and $S(x) \subseteq x$. We show that the family of homogeneous sets (with respect to a partition of a front) as given by the Nash-Williams' theorem admits such a Borel selector. However, we also show that the analogous result for Galvin's lemma is not true by proving that there is an $F_{\sigma}$ tall ideal on $\mathbb{N}$ without a Borel selector. The proof is not constructive since it is based on complexity considerations. We construct a $\Pi_{2}^{1}$ tall ideal on $\mathbb{N}$ without a tall closed subset.
\end{abstract}

§1. Introduction. A family $\mathcal{C}$ of subsets of $\mathbb{N}$ is tall if for every infinite $x \subseteq \mathbb{N}$ there is an infinite $y \in \mathcal{C}$ such that $y \subseteq x$. We are interested in tall families $\mathcal{C}$ which are in addition definable as subsets of $2^{\mathbb{N}}$. Take for example the set hom $(c)$ of all monochromatic subsets of $\mathbb{N}$ for some coloring $c:[\mathbb{N}]^{2} \rightarrow 2$. This is, by Ramsey theorem, a tall family and moreover it is a closed subset of $2^{\mathbb{N}}$. We deal with the question of when we can effectively witness that a family is tall, i.e., when there is a Borel function $S: 2^{\mathbb{N}} \rightarrow 2^{\mathbb{N}}$ such that for every infinite $x \in 2^{\mathbb{N}}, S(x) \in \mathcal{C}, S(x)$ is infinite and $S(x) \subseteq x$. We call such a function $S$ a Borel selector for $\mathcal{C}$. Note that if there is a Borel selector $S$ for $\mathcal{C}$, then $\mathcal{C}$ contains an analytic subfamily which is also tall. This leads to a natural basis problem of whether a given tall family $\mathcal{C}$ contains a simpler tall subfamily $\mathcal{C}^{\prime} \subseteq \mathcal{C}$. By simpler we mean that $\mathcal{C}^{\prime}$ is of lower complexity (for example closed) or is of a specific form (for example hom $(c)$ for some coloring $c$ ).

An important source of examples of tall families are tall Borel ideals on $\mathbb{N}$. Up to now, all known examples of Borel tall ideals (see, for instance, $[6,7]$ ) have a Borel selector (see Section 3.3). One of the main results of this article is the existence of an $F_{\sigma}$ tall ideal without a Borel selector. The proof of this result is based on the following facts. Every $F_{\sigma}$ ideal can be coded by a closed collection of sets, i.e., by an element of the hyperspace $K\left(2^{\mathbb{N}}\right)$. In [5] it is proved that the set of codes of tall $F_{\sigma}$ ideals is a $\Pi_{2}^{1}$-complete subset of $K\left(2^{\mathbb{N}}\right)$. To show that there is an $F_{\sigma}$ ideal without a selector we prove that the complexity of the set of codes of $F_{\sigma}$ ideals with a Borel selector is $\Sigma_{2}^{1}$. However, it is an open question to find a concrete example of such $F_{\sigma}$ ideal. This result is a generalization of the classical fact that there is a closed subset of $\mathbb{N}^{\mathbb{N}} \times \mathbb{N}^{\mathbb{N}}$ whose projection is $\mathbb{N}^{\mathbb{N}}$ but without a Borel uniformization (see Corollary 4.19).

Received February 22, 2018.

2010 Mathematics Subject Classification. Primary 03E15, 03 E05.

Key words and phrases. Borel ideals, tall families, Borel selector, Galvin's Lemma.

(C) 2019, Association for Symbolic Logic $0022-4812 / 19 / 8401-0016$ DOI: $10.1017 /$ jsl.2018.66 
Another important class of tall families are the collection of homogeneous sets with respect to a partition of $[\mathbb{N}]^{\omega}$, the infinite subsets of $\mathbb{N}$. Given $\mathcal{O} \subseteq[\mathbb{N}]^{\omega}$, a set $x \subseteq \mathbb{N}$ is called $\mathcal{O}$-homogeneous, if either $[x]^{\omega} \subseteq \mathcal{O}$ or $[x]^{\omega} \cap \mathcal{O}=\emptyset$. A well known theorem of Silver [12] says that for every analytic subset $\mathcal{O}$ of $[\mathbb{N}]^{\omega}$ the collection $\operatorname{hom}(\mathcal{O})$ of $\mathcal{O}$-homogeneous sets is tall. When $\mathcal{O}$ is open (resp. clopen), the corresponding Ramsey result is called Galvin's lemma [3] (resp. Nash-Williams' theorem [11]). The existence of Borel selectors for families of the form $\operatorname{hom}(\mathcal{O})$ may be interpreted as the fact that the corresponding Ramsey theorem holds uniformly, i.e., there is a Borel (uniform, definable) way to compute, given $x \in[\mathbb{N}]^{\omega}$, an infinite $\mathcal{O}$-homogeneous subset of $x$. For instance, the fact that the Random ideal $\mathcal{R}[6]$ has a Borel selector is due to the fact that there is uniform approach of finding an infinite monochromatic subset of a given set $x \subseteq \mathbb{N}$, which could be seen as having a "Borel proof" of Ramsey's theorem [7]. Analogously, we show that Nash-Williams' theorem also has a uniform version and thus $\operatorname{hom}(\mathcal{O})$ has a Borel selector for every clopen set $\mathcal{O}$. However, we show there is an open set $\mathcal{O}$ such that $\operatorname{hom}(\mathcal{O})$ does not have a Borel selector and therefore Galvin's lemma does not admit a uniform version.

Ramsey -type theorems have been analyzed from a related but different complexity point of view. Solovay ([14]) showed that if $\mathcal{O} \subseteq[\mathbb{N}]^{\omega}$ is open and $[x]^{\omega} \subseteq \mathcal{O}$ for every $x \in \operatorname{hom}(\mathcal{O})$, then hom $(\mathcal{O})$ contains an element which is hyperarithmetical in the code of $\mathcal{O}$ (see also [1]).

Finally, we show that the basis problem also has a negative answer. We construct a $\Pi_{2}^{1}$ tall ideal $\mathcal{I}$ such that $\operatorname{hom}(\mathcal{O}) \nsubseteq \mathcal{I}$ for all open set $\mathcal{O} \subseteq[\mathbb{N}]^{\omega}$, in particular, $\mathcal{I}$ does not contain any tall closed subset. It is still an open question whether every tall Borel (analytic) ideal contains a closed tall subset.

§2. Preliminaries. In this section we fix our notation, give some basic definitions and results that are later used. We consider the natural isomorphism $\mathcal{P}(\mathbb{N}) \approx 2^{\mathbb{N}}$ and view all relations and operation such as $\subseteq, \cap, \cup, \triangle$, [-] $]^{<\omega}$, etc, as defined on $2^{\mathbb{N}}$ i.e., we use $x \subseteq y, x \cap y$, [x] $]^{<\omega}$, etc, for $x, y \in 2^{\mathbb{N}}$. For $x$ a subset of $\mathbb{N}$ and $n \in \mathbb{N}$ we let $x / n=\{m \in x: n<m\}$. We use the standard descriptive set theoretic notions and notation (as in [8]). The projective classes are denoted $\Sigma_{n}^{1}$ and $\boldsymbol{\Pi}_{n}^{1}$.

Definition 2.1. Let $\mathcal{C} \subseteq 2^{\mathbb{N}}$ be a tall family. We say that $\mathcal{C}$ has a Borel selector, if there is a Borel function $S: 2^{\mathbb{N}} \rightarrow 2^{\mathbb{N}}$ such that for every infinite set $x \in 2^{\mathbb{N}}, S(x)$ is infinite, $S(x) \subseteq x$ and $S(x) \in \mathcal{C}$.

Note that we define the notion of a Borel selector only for tall families so if we say that $\mathcal{C}$ has a Borel selector it automatically means that $\mathcal{C}$ is tall. We say that a family $\mathcal{C}$ is hereditary if $y \in \mathcal{C}$ whenever there is $x \in \mathcal{C}$ such that $y \subseteq x$. We say that $\mathcal{I} \subseteq 2^{\mathbb{N}}$ is an ideal on $\mathbb{N}$ if it is hereditary and it is closed under finite unions. As usual, we define $\mathcal{I}^{+}$as $2^{\mathbb{N}} \backslash \mathcal{I}$.

The following characterization of an $F_{\sigma}$ ideal on $\mathbb{N}$ was given by Mazur [10]. Recall that a map $\varphi: 2^{\mathbb{N}} \rightarrow[0, \infty]$ is a lower-semicontinuous submeasure (lscsm) if for all $x, y \in \mathbb{N}$

- $\varphi(\emptyset)=0$,

- $x \subseteq y$ implies $\varphi(x) \leq \varphi(y)$ 
- $\varphi(x \cup y) \leq \varphi(x)+\varphi(y)$,

- $\varphi(x)=\lim _{n \rightarrow \infty} \varphi(x \cap n)$.

Each $1 \operatorname{scsm} \varphi$ naturally corresponds to the $F_{\sigma}$ ideal $\operatorname{Fin}(\varphi):=\{x: \varphi(x)<\infty\}$.

THEOREM 2.2 (Mazur [10]). An ideal $\mathcal{I}$ is $F_{\sigma}$ if and only if there is lscsm $\varphi$ such that $\mathcal{I}=\operatorname{Fin}(\varphi)$.

From this characterization one easily deduces (see for example [5]) the following result which allows us to consider $K\left(2^{\mathbb{N}}\right)$, the hyperspace of closed subsets of $2^{\mathbb{N}}$ endowed with its usual metric topology, as a space of codes of $F_{\sigma}$ ideals. For $K \in K\left(2^{\mathbb{N}}\right)$, let $\mathcal{I}_{K}$ be ideal generated by $K$, i.e., $x \in \mathcal{I}_{K}$ if and only if there are $y_{0}, \ldots, y_{n-1} \in K$ such that $x \subseteq \bigcup_{i<n} y_{i}$. Clearly, $\mathcal{I}_{K}$ is $F_{\sigma}$.

Proposition 2.3. For every $F_{\sigma}$ ideal $\mathcal{I}$ there is $K \in K\left(2^{\mathbb{N}}\right)$ such that $\mathcal{I}=\mathcal{I}_{K}$.

Proof. We include the proof given in [5] for the sake of completness. Let $\operatorname{supp}(\mathcal{I})=\{n \in \mathbb{N}:\{n\} \in \mathcal{I}\}$ and $\mathcal{I}=\bigcup_{n} K_{n}$ where each $K_{n}$ is closed. Enumerate $\operatorname{supp}(\mathcal{I})$ as $\left\{a_{n}: n \in \mathbb{N}\right\}$. Define a tree $T=\bigcup_{n} T_{n}$ on $2^{<\omega}$ where $y \in\left[T_{n}\right]$ if $y(i)=0$ for every $i<a_{n}, y\left(a_{n}\right)=1$ and there is $x \in K_{n}$ such that $y(i)=x(i)$ for every $i>a_{n}$. Note that now for every $x \in \mathcal{I}$ there is $y \in[T]$ such that $x \Delta y$ is finite and therefore if we put $K=[T]$ we have $\mathcal{I}_{K}=\mathcal{I}$.

Let $\mathcal{T}$ be the collection of all $K \in K\left(2^{\mathbb{N}}\right)$ such that $\mathcal{I}_{K}$ is tall. The following result is crucial for our purposes.

THEOREM 2.4 ([5]). $\mathcal{T}$ is $\boldsymbol{\Pi}_{2}^{1}$-complete subset of $K\left(2^{\mathbb{N}}\right)$.

We present the original proof from [5] for the convenience of the reader. The proof uses a special version of a result of Becker, Kahane, and Louveau (see [2]). Consider the following set $\mathcal{U} \subseteq\left(C\left(2^{\mathbb{N}} \times 2^{\mathbb{N}}, 2\right)\right)^{\omega} \times 2^{\mathbb{N}}$ given by

$\left(\left(f_{n}\right)_{n \in \omega}, x\right) \in \mathcal{U} \Leftrightarrow$ there is $z \subseteq \mathbb{N}$ such that $\left(f_{n}\left(x,{ }_{-}\right)\right)_{n \in z}$ converges pointwise to 0 .

THEOREM 2.5 ([8], Theorem 37.14). The set $\mathcal{U}$ is $\left(C\left(2^{\mathbb{N}} \times 2^{\mathbb{N}}, 2\right)\right)^{\omega}$-universal for $\Sigma_{2}^{1}\left(2^{\mathbb{N}}\right)$, i.e., every $\Sigma_{2}^{1}$ subset of $2^{\mathbb{N}}$ appears as a section of $\mathcal{U}$.

Proof of Theorem 2.4. Let $X \subseteq 2^{\mathbb{N}}$ be $\Sigma_{2}^{1}$ set. By the universality of $\mathcal{U}$, there is $\left(f_{n}\right)_{n \in \omega} \in\left(C\left(2^{\mathbb{N}} \times 2^{\mathbb{N}}, 2\right)\right)^{\omega}$ such that $X=\left\{x:\left(\left(f_{n}\right)_{n \in \omega}, x\right) \in \mathcal{U}\right\}$. Define a map $\sigma: 2^{\mathbb{N}} \rightarrow K\left(2^{\mathbb{N}}\right)$ by $y \in \sigma(x)$ if and only if there is $z \in 2^{\mathbb{N}}$ such that $y(n)=f_{n}(x, z)$ for all $n \in \mathbb{N}$. This function is clearly continuous. To finish the proof, it suffices to show that $x \in X$ if and only if $\mathcal{I}_{\sigma(x)}$ is not tall.

Given $x \in X$, there is $z \subseteq \mathbb{N}$ such that $\left(f_{n}(x,-)\right)_{n \in z}$ converges pointwise to 0 , that is $z \cap y$ is finite for every $y \in \sigma(x)$. Thus, $z$ does not contain any infinite set in $\mathcal{I}_{\sigma(x)}$. Hence $\mathcal{I}_{\sigma(x)}$ is not tall.

On the other hand, suppose $x \notin X$. To show that $\mathcal{I}_{\sigma(x)}$ is tall, fix $z \subseteq \mathbb{N}$ infinite. Since $\left(\left(f_{n}\right)_{n \in \omega}, x\right) \notin \mathcal{U}$, then $\left(f_{n}(x,-)\right)_{n \in z}$ does not converge pointwise to 0 . Therefore there is a $y \in \sigma(x)$ such that $z \cap y$ is infinite, so $\mathcal{I}_{\sigma(x)}$ is tall.

To finish the proof it is enough to realize that the set $\mathcal{T}$ is $\boldsymbol{\Pi}_{2}^{1}$. But this follows easily from the definition of $\mathcal{T}$ :

$$
\mathcal{T}=\left\{K \in K\left(2^{\mathbb{N}}\right): \forall x \in 2^{\mathbb{N}} \backslash \text { Fin } \exists y \in K \backslash \text { Fin }|y \cap x|=\omega\right\} .
$$

Next, we state the combinatorial theorems (as presented in [15]). Let $s \in[\mathbb{N}]^{<\omega}$ and $t \in[\mathbb{N}] \leq \omega$. We write $s \sqsubseteq t$ when there is $n \in \mathbb{N}$ such that $s=t \cap\{0,1, \ldots, n\}$ and we say that $s$ is an initial segment of $t$. 
TheOREM 2.6 (Galvin). Let $\mathcal{F} \subseteq[\mathbb{N}]^{<\omega}$ and an infinite $x \in 2^{\mathbb{N}}$. Then there is an infinite $y \subseteq x$ such that one of the following holds

- for all $z \in[y]^{\omega}$ there is $s \in \mathcal{F}$ such that $s \sqsubseteq z$,

- $[y]^{<\omega} \cap \mathcal{F}=\emptyset$.

We can think of $\mathcal{F}$ as a coloring of $[\mathbb{N}]^{<\omega}$ and put $\operatorname{hom}(\mathcal{F}) \subseteq 2^{\mathbb{N}}$ for the family of all $y$ that satisfy one of the conditions in the conclusion of Galvin's theorem, such sets are called $\mathcal{F}$-homogeneous. It is clear that $\operatorname{hom}(\mathcal{F})$ is an hereditary tall collection. Moreover, the family of all sets that satisfy the second condition is closed and the family of sets that satisfy the first condition is $\Pi_{1}^{1}$. We write $\mathbb{P}_{2}$ for the set of all $\mathcal{F} \subseteq[\mathbb{N}]^{<\omega}$ such that for every infinite $x \in 2^{\mathbb{N}}$ there is an infinite $y \subseteq x$ such that $[y]^{<\omega} \cap \mathcal{F}=\emptyset$, i.e., the first condition in the conclusion of Galvin's theorem is never satisfied.

A special type of coloring of $[\mathbb{N}]^{<\omega}$ is as follows. We say that $\mathcal{B} \subseteq[\mathbb{N}]^{<\omega}$ is a front on an infinite set $x \in 2^{\mathbb{N}}$ if

- every two elements of $\mathcal{B}$ are $\sqsubseteq$-incomparable,

- every infinite $y \subseteq x$ has an initial segment in $\mathcal{B}$.

Theorem 2.7 (Nash-Williams). Let $\mathcal{B}$ be a front on $\mathbb{N}$ and $\mathcal{F} \subseteq \mathcal{B}$. Then for every infinite $x \in 2^{\mathbb{N}}$ there is an infinite $y \subseteq x$ such that one of the following holds

- $[y]^{<\omega} \cap \mathcal{B} \subseteq \mathcal{F}$,

- $[y]^{<\omega} \cap \mathcal{F}=\emptyset$.

Let $\mathcal{F} \subseteq \mathcal{B}$ as above, it is easy to verify that $y \in \operatorname{hom}(\mathcal{F})$ if and only if $y$ satisfies one of the conditions from the Nash-Williams' theorem. Moreover, the family $\operatorname{hom}(\mathcal{F})$ is easily seen to be closed, hereditary, and tall.

Proposition 2.8. For every closed, tall, and hereditary $K \subseteq 2^{\mathbb{N}}$ there is $\mathcal{F} \subseteq[\mathbb{N}]^{<\omega}$ such that $\operatorname{hom}(\mathcal{F})=K$.

Proof. Define $\mathcal{F}_{K}=\left\{s \in[\mathbb{N}]^{<\omega}: s \notin K\right\}$. We claim that $\operatorname{hom}\left(\mathcal{F}_{K}\right)$ is equal to $\left\{y \in[\mathbb{N}]^{\omega}:[y]^{<\infty} \cap \mathcal{F}_{K}=\emptyset\right\}$. Let $y \in \operatorname{hom}\left(\mathcal{F}_{K}\right)$ and suppose, towards a contradiction, that $y$ satisfies the first condition in the conclusion of Galvin's theorem. Since $K$ is tall there is an infinite $z \subseteq y$ such that $z \in K$. As $y$ satisfies the first condition, there is $s \in \mathcal{F}_{K}$ such that $s \sqsubseteq z$ but since $K$ is hereditary we have $s \in K$ and this contradicts the definition of $\mathcal{F}_{K}$.

It remains to check that $K=\operatorname{hom}\left(\mathcal{F}_{K}\right)$. Clearly $\subseteq$ holds. Conversely, let $x \notin K$, since $K$ is hereditary and closed there must be some $n \in \mathbb{N}$ such that $x \cap n \notin K$ then we have $x \cap n \in \mathcal{F}_{K}$. Thus $x \notin \operatorname{hom}\left(\mathcal{F}_{K}\right)$.

Proposition 2.9. The set $\mathbb{P}_{2}$ is $\boldsymbol{\Pi}_{2}^{1}$-complete.

Proof. This is a generalization of previous argument. It is easily seen that $\mathbb{P}_{2}$ is $\Pi_{2}^{1}$. Thus, it suffices to find a continuous map $\psi: K\left(2^{\mathbb{N}}\right) \rightarrow 2^{[\mathbb{N}]^{<\omega}}$ such that $K \in \mathcal{T}$ if and only if $\psi(K) \in \mathbb{P}_{2}$, where $\mathcal{T}$ is as in Theorem 2.4. Consider the map $\psi: K\left(2^{\mathbb{N}}\right) \rightarrow 2^{[\mathbb{N}]^{<\omega}}$ given by

$$
s \in \psi(K) \Leftrightarrow \forall x \in K s \nsubseteq x .
$$

We show first that $\psi$ is continuous. Let $s \in[\mathbb{N}]^{<\omega}$ and consider the subbasic clopen set $\mathcal{O}=\left\{\mathcal{F} \in 2^{[\mathbb{N}]^{<\omega}}: s \in \mathcal{F}\right\}$. Then

$$
\psi(K) \notin \mathcal{O} \Leftrightarrow \exists x \in 2^{\mathbb{N}}(x \in K \wedge s \subseteq x) .
$$


This is clearly a projection of a compact subset of $2^{\mathbb{N}} \times K\left(2^{\mathbb{N}}\right)$. Thus $\psi^{-1}(\mathcal{O})$ is open. We have the same conclusion for the other subbasic clopen set $\mathcal{O}=\{\mathcal{F} \in$ $\left.2^{[\mathbb{N}]^{<\omega}}: s \notin \mathcal{F}\right\}$.

Let $K \in \mathcal{T}$. To prove that $\psi(K) \in \mathbb{P}_{2}$ we need to show that $h o m(\psi(K))=\{y \in$ $\left.[\mathbb{N}]^{\omega}:[y]^{<\infty} \cap \psi(K)=\emptyset\right\}$. In fact, as $\mathcal{I}_{K}$ is tall, for every infinite $y$ there is $z \in K$ such that $z \cap y$ is infinite. Using this fact and an argument similar to that in the proof of Proposition 2.8, we get that there is no element of $\operatorname{hom}\left(\mathcal{F}_{K}\right)$ satisfying the first condition in the conclusion of Galvin's theorem.

Conversely, let $K \notin \mathcal{T}$. As $\mathcal{I}_{K}$ is not tall, there is an infinite $x \in 2^{\mathbb{N}}$ such that $x \cap y$ is finite for all $y \in K$. By Galvin's Lemma, we can assume w.l.o.g. that $x \in \operatorname{hom}(\psi(K))$. We show that $x$ satisfies the first condition in the conclusion of Galvin's theorem, that is, $\psi(K) \notin \mathbb{P}_{2}$. Suppose, towards a contradiction, that $[x]^{<\infty} \cap \psi(K)=\emptyset$. Then for all $n$, there is $y_{n} \in K$ such that $x \cap n \subseteq y_{n}$. Since $K$ is compact, there is $z \in K$ such that $x \subseteq z$ but this is imposible as $x \cap z$ is finite.

§3. Positive results. In this section we prove the uniform version of the NashWilliams's theorem. To state our theorem in full generality we must first introduce several definitions.

3.1. Uniformly $p^{+}, q^{+}$and selective ideals. Let $\mathcal{I}$ be an ideal on $\mathbb{N}$. We say that $\mathcal{I}$ is $q^{+}$if for all $x \in \mathcal{I}^{+}$and every partition $\left\{s_{n}\right\}_{n}$ of $x$ into finite sets there is $y \subseteq x$ such that $y \in \mathcal{I}^{+}$and $\left|y \cap x_{n}\right| \leq 1$ for all $n \in \mathbb{N}$. A subset of $\bigcup s_{i}$ is a selector for $\left\{s_{n}\right\}_{n}$ if it selects at most one point of each piece $s_{n}$. The ideal $\mathcal{I}$ is $p^{+}$if for every decreasing sequence $\left(x_{n}\right)_{n}$ of sets in $\mathcal{I}^{+}$there is $x \in \mathcal{I}^{+}$such that $x \backslash x_{n}$ is finite for all $n$. It is selective, if for every decreasing sequence $\left(x_{n}\right)_{n}$ of sets in $\mathcal{I}^{+}$there is $x \in \mathcal{I}^{+}$such that $x / n \subseteq x_{n}$ for all $n \in x$. We are interested in the uniform versions of these notions. We say that a Borel ideal $\mathcal{I}$ is uniformly selective if there is a Borel function $F$ from $\left(2^{\mathbb{N}}\right)^{\mathbb{N}}$ into $2^{\mathbb{N}}$ such that whenever $\left(x_{n}\right)_{n}$ is a decreasing sequence of sets in $\mathcal{I}^{+}$, then $x=F\left(\left(x_{n}\right)_{n}\right)$ is in $\mathcal{I}^{+}$and $x / n \subseteq x_{n}$ for all $n \in x$. In an analogous way, we define when an ideal is uniformly $p^{+}$. We say that $\mathcal{I}$ is uniformly $q^{+}$, if there is a Borel function $F$ from $\left(2^{\mathbb{N}}\right)^{\mathbb{N}}$ into $2^{\mathbb{N}}$ such that whenever $\left\{s_{n}\right\}_{n}$ is a partition of a set $x$ in $\mathcal{I}^{+}$into finite sets, then $y=F\left(\left(s_{n}\right)_{n}\right) \subseteq x, y$ belongs to $\mathcal{I}^{+}$and $\left|y \cap s_{n}\right| \leq 1$ for all $n$.

Let $\mathcal{I}$ be a Borel ideal. Consider the collection $\mathcal{P}$ of all $\left(x_{n}\right)_{n}$ in $\left(2^{\mathbb{N}}\right)^{\mathbb{N}}$ such that $\left(x_{n}\right)_{n}$ is decreasing and each $x_{n}$ belongs to $\mathcal{I}^{+}$. Clearly $\mathcal{P}$ is a Borel subset of $\left(2^{\mathbb{N}}\right)^{\mathbb{N}}$. Thus it suffices to define on $\mathcal{P}$ the Borel function required to show uniform $p^{+}$or uniform selectivity. Analogously, let $\mathcal{Q}$ be the collection of all $\left(s_{n}\right)_{n}$ in $\left(2^{\mathbb{N}}\right)^{\mathbb{N}}$ such that $\left(s_{n}\right)_{n}$ is pairwise disjoint, each $s_{n}$ is finite and $\bigcup_{n} s_{n} \in \mathcal{I}^{+}$. Then $\mathcal{Q}$ is a Borel subset of $\left(2^{\mathbb{N}}\right)^{\mathbb{N}}$. Thus, it suffices to define on $\mathcal{Q}$ the Borel function required to have uniform $q^{+}$.

LEMma 3.1. A Borel ideal $\mathcal{I}$ is uniformly selective if and only if it is uniformly $p^{+}$ and $q^{+}$.

Proof. It is known that an ideal is selective if and only if it is $p^{+}$and $q^{+}$(see for instance [16, Lemma 7.4]). We will verify that the standard proof of this result is effective. Let $\mathcal{I}$ be an uniformly selective Borel ideal. It is clear that $\mathcal{I}$ is uniformly $p^{+}$. Let us check that it is uniformly $q^{+}$. As we explain above, it suffices to find a function $F$, as in the definition of an uniformly $q^{+}$ideal, which is defined only on $\mathcal{Q}$. 
Consider the function $G$ from $\mathcal{Q}$ into $\left(2^{\mathbb{N}}\right)^{\mathbb{N}}$ given by $G\left(\left(s_{n}\right)_{n}\right)=\left(x_{n}\right)_{n}$ where $x_{n}=\bigcup\left\{s_{j}:\{0, \ldots, n\} \cap s_{j}=\emptyset\right\}$ for each $n$. It is clear that each $x_{n} \in \mathcal{I}^{+}$and $G$ is Borel. Observe that if $G\left(\left(s_{n}\right)_{n}\right)=\left(x_{n}\right)_{n}$ and $y$ is any diagonalization of $\left(x_{n}\right)_{n}$ (i.e., $y / n \subseteq x_{n}$ for all $\left.n \in y\right)$, then $\left|y \cap s_{i}\right| \leq 1$ for all $i$. Let $H$ be a Borel function as in the definition of uniform selectivity. Then $F=H \circ G$ works.

Suppose now that $\mathcal{I}$ is uniformly $p^{+}$and $q^{+}$. We will show that $\mathcal{I}$ is uniformly selective. As before, it suffices to define on $\mathcal{P}$ the Borel function required to show uniform selectivity. Let $\left(x_{n}\right)_{n}$ be a sequence in $\mathcal{P}$. By the property $p^{+}$, there is $y \in \mathcal{I}^{+}$ such that $y \subseteq \subseteq^{*} x_{n}$ for all $n$. Let $\left(n_{k}\right)_{k}$ be a strictly increasing sequence of natural numbers such that $n_{0}=0$ and $y \backslash x_{n_{k}} \subseteq\left\{0, \ldots, n_{k+1}\right\}$ for all $k$. For instance, let $n_{k+1}=\max \left\{\max \left(y \backslash x_{n_{k}}\right), n_{k}\right\}+1$. By the property $q^{+}$, there is $z \in \mathcal{I}^{+}$such that $z \subseteq y$ and $\left|z \cap\left[n_{k}, n_{k+1}\right)\right| \leq 1$ for all $k$ (where $\left[n_{k}, n_{k+1}\right.$ ) is the interval of all $n$ in $\mathbb{N}$ such that $\left.n_{k} \leq n<n_{k+1}\right)$. Let $z_{0}$ be the union of all sets $z \cap\left[n_{k}, n_{k+1}\right)$ with $k$ even and $z_{1}$ be the union of all sets $z \cap\left[n_{k}, n_{k+1}\right)$ with $k$ odd. Either $z_{0}$ or $z_{1}$ (it could be both) belongs to $\mathcal{I}^{+}$. Thus, we can assume w.l.o.g that $z \cap\left[n_{2 k}, n_{2 k+1}\right)=\emptyset$ for all $k$ (or $z \cap\left[n_{2 k+1}, n_{2 k+2}\right.$ ) $=\emptyset$ for all $k$ ). It is easy to verify that $z / n \subseteq x_{n}$ for all $n \in z$. Thus, $\mathcal{I}$ is selective. To see that this proof is effective just observe that the set $y$ is choosen using the Borel function given by the fact that $\mathcal{I}$ is uniformly $p^{+}$. Now the sequence $\left(n_{k}\right)_{k}$ was choosen in a Borel way as a function of $y$ and $\left(x_{n}\right)_{n}$. Then we use the Borel function given by the uniform $q^{+}$property to select the set $z$. Finally, since $\mathcal{I}$ is Borel, we can pick in a Borel way among $z_{0}$ and $z_{1}$ the appropriated alternative.

TheOREM 3.2. Let $\mathcal{I}$ be a $F_{\sigma}$ ideal. Then,

(i) $\mathcal{I}$ is uniformly $p^{+}$.

(ii) if $\mathcal{I}$ is $q^{+}$, then it is uniformly $q^{+}$.

In particular, every selective $F_{\sigma}$ ideal is uniformly selective.

Proof. Let $\left\{s_{k}\right\}_{k}$ be an enumeration of $[\mathbb{N}]^{<\omega}$ and let $\mu$ be the lower semicontinuous submeasure such that $\mathcal{I}=\left\{x \in 2^{\mathbb{N}}: \mu(x)<\infty\right\}$. First we claim that for each $n \in \mathbb{N}$ there is a Borel function $G_{n}: 2^{\mathbb{N}} \rightarrow 2^{\mathbb{N}}$ such that for all $x \notin \mathcal{I}, G_{n}(x)$ is a finite subset of $x$ and $\mu\left(G_{n}(x)\right) \geq n$. Define $G_{n}(x)=\emptyset$ for $x \in \mathcal{I}$. For $x \in \mathcal{I}^{+}$let $G_{n}(x)=s_{k}$ where $k$ is the minimal index such that $s_{k} \subseteq x$ and $\mu\left(s_{k}\right) \geq n$.

We will define the Borel selection functions on the collections $\mathcal{P}$ and $\mathcal{Q}$ as it was explained above.

(i) Let $\left(x_{n}\right)_{n}$ be a sequence in $\mathcal{P}$. Define $G\left(\left(x_{n}\right)_{n}\right)=\bigcup_{n} G_{n}\left(x_{n}\right)$. Then $G$ is Borel and has the required property since, letting $y=G\left(\left(x_{n}\right)_{n}\right)$, we have that $y \backslash x_{n} \subseteq G\left(x_{0}\right) \cup \cdots \cup G\left(x_{n-1}\right)$ is a finite set.

(ii) We define recursively Borel functions $F_{n}: \mathcal{Q} \rightarrow 2^{\mathbb{N}}$ and $K_{n}: \mathcal{Q} \rightarrow \mathbb{N}$ for all $n \in \mathbb{N}$. For $\left(t_{i}\right)_{i} \in \mathcal{Q}$ we define

- $F_{1}\left(\left(t_{i}\right)_{i}\right)=s_{k}$ where $k$ is the smallest $j$ such that $\mu\left(s_{j}\right) \geq 1$ and $s_{j}$ is a (finite) selector for $\left(t_{i}\right)_{i}$. Such $j$ exists since $\mathcal{I}$ is $q^{+}$. In fact, let $y$ be a selector for $\left(t_{i}\right)_{i}$ such that $y \in \mathcal{I}^{+}$. Hence, $G_{1}(y)$ is a finite selector for $\left(t_{i}\right)_{i}$ as requiered. Let $K_{1}\left(\left(t_{i}\right)_{i}\right)$ be the smallest $k$ such that $F_{1}\left(\left(t_{i}\right)_{i}\right) \cap t_{j}=\emptyset$ for all $j \geq k$.

- $F_{n+1}\left(\left(t_{i}\right)_{i}\right)=s_{k}$ where $k$ is the smallest $j$ such that $\mu\left(s_{j}\right) \geq n+1$ and $s_{j}$ is a (finite) selector for $\left(t_{i}\right)_{i \geq k_{n}}$ where $k_{n}=K_{n}\left(\left(t_{i}\right)_{i}\right)$. As before, as 
$\mathcal{I}$ is $q^{+}$, such $k$ exists. Let $K_{n+1}\left(\left(t_{i}\right)_{i}\right)$ be the smallest $k>k_{n}$ such that $F_{n+1}\left(\left(t_{i}\right)_{i}\right) \cap t_{j}=\emptyset$ for all $j \geq k$.

Then $F_{n}$ and $K_{n}$ are Borel functions for all $n$. Finally put $F\left(\left(t_{i}\right)_{i}\right)=$ $\bigcup_{n \in \mathbb{N}} F_{n}\left(\left(t_{i}\right)_{i}\right)$. From the construction it is clear that $y=F\left(\left(t_{i}\right)_{i}\right)$ is a selector for $\left(t_{i}\right)_{i}$ and $\mu(y)=\infty$, hence $y \in \mathcal{I}^{+}$.

COROllary 3.3. Fin is uniformly selective.

Recall that $\mathcal{A} \subseteq[\mathbb{N}]^{\omega}$ is an almost disjoint family if $x \cap y \in$ Fin for every $x, y \in \mathcal{A}$ with $x \neq y$. Let $\mathcal{A}$ be an almost disjoint family and $\mathcal{I}(\mathcal{A})$ be the ideal generated by $\mathcal{A}$. By a result of Mathias [9], $\mathcal{I}(\mathcal{A})$ is selective. It is easy to verify that when $\mathcal{A}$ is closed (as a subset of $2^{\mathbb{N}}$ ), then $\mathcal{I}(\mathcal{A})$ is $F_{\sigma}$. Hence, from Theorem 3.2 we get the following

Corollary 3.4. Let $\mathcal{A}$ be a closed almost disjoint family. Then $\mathcal{I}(\mathcal{A})$ is uniformly selective.

The previous result naturally suggests the following.

QUESTION 3.5. Is $\mathcal{I}(\mathcal{A})$ uniformly selective for every almost disjoint Borel family $\mathcal{A}$ ? More generally, is every Borel selective ideal uniformly selective?

3.2. Uniform Ramsey-type theorems. Recall that the lexicographic order $<_{\text {lex }}$ on $[\mathbb{N}]^{<\omega}$ is defined by $s<_{\text {lex }} t$ if $\min (s \triangle t) \in s$. Let $x \in 2^{\mathbb{N}}$ be infinite and $\mathcal{B} \subseteq[x]^{<\omega}$ be a front on $x$. Then the restriction of $<_{\text {lex }}$ on $\mathcal{B}$ is a well-order and its order type is called the rank of $\mathcal{B}($ denoted $\operatorname{rank}(\mathcal{B}))$.

For $\mathcal{F} \subseteq[\mathbb{N}]^{<\omega}$ we define $\overline{\mathcal{F}}=\left\{s \in[\mathbb{N}]^{<\omega}: s \sqsubseteq t\right.$ for some $\left.t \in \mathcal{F}\right\}$.

LEMma 3.6. Let $\mathcal{B}$ be a front and $\mathcal{F} \subseteq \overline{\mathcal{B}}$. Let $\widehat{\mathcal{F}}=\left\{s \in[\mathbb{N}]^{<\omega}: \exists t \in \mathcal{F}, \exists t^{\prime} \in\right.$ $\left.\mathcal{B}, t \sqsubseteq s \sqsubseteq t^{\prime}\right\}$. Then $x \in \operatorname{hom}(\mathcal{F})$ if and only if $[x]^{<\omega} \cap \mathcal{F}=\emptyset$ or $[x]^{<\omega} \cap \overline{\mathcal{B}} \subseteq \overline{\mathcal{F}} \cup \widehat{\mathcal{F}}$.

Proof. Let $x \in \operatorname{hom}(\mathcal{F})$. Suppose the first item in the conclusion of Theorem 2.6 holds. Let $s \subset x$ with $s \in \overline{\mathcal{B}}$ and put $y=s \cup\{n \in x: n>\max s\}$. Thus there is $t \in \mathcal{F}$ such that $t \sqsubset y$. Hence, $s \sqsubseteq t$ or $t \sqsubseteq s$. In either case, $s \in \overline{\mathcal{F}} \cup \widehat{\mathcal{F}}$. Conversely, suppose that $[x]^{<\omega} \cap \overline{\mathcal{B}} \subseteq \overline{\mathcal{F}} \cup \widehat{\mathcal{F}}$ and let $y \in[x]^{<\omega}$. Since $\mathcal{B}$ is a front, there is $t \in \mathcal{B}$ such that $t \sqsubset y$. Then $t \in \overline{\mathcal{F}} \cup \widehat{\mathcal{F}}$. Since $t \in \mathcal{B}$, there is $s \sqsubseteq t$ with $s \in \mathcal{F}$. Hence, $x \in \operatorname{hom}(\mathcal{F})$.

THEOREM 3.7. Let $\mathcal{I}$ be a uniformly selective Borel ideal on $\mathbb{N}$ and let $\mathcal{B}$ be a front on some set $z \in \mathcal{I}^{+}$. There is a Borel map $S: 2^{\overline{\mathcal{B}}} \times\left(\mathcal{I}^{+}\lceil z) \rightarrow \mathcal{I}^{+}\right.$such that $S(\mathcal{F}, x)$ is a $\mathcal{F}$-homogeneous subset of $x$ for all $x \in \mathcal{I}^{+}\lceil z$.

Proof. Let $\mathcal{B}$ be a front on $z$ and proceed by induction on $\alpha=\operatorname{rank}(\mathcal{B})$. If $\operatorname{rank}(\mathcal{B})=\omega$, then $\mathcal{B}=[z]^{1}$. Let $S(\mathcal{F}, x)=(\bigcup \mathcal{F}) \cap x$, if $(\bigcup \mathcal{F}) \cap x \in \mathcal{I}^{+}$. Otherwise, $S(\mathcal{F}, x)=x \backslash \cup \mathcal{F}$. Since $\mathcal{I}^{+}$is Borel, then $S$ is a Borel function.

Now suppose that the claim holds for all fronts on any $z \in \mathcal{I}^{+}$of rank less than $\alpha$. Let $\mathcal{B}$ be a front on $z \in \mathcal{I}^{+}$of rank $\alpha$. For each $n \in \mathbb{N}$ and $\mathcal{F} \subseteq \mathcal{B}$, let

$$
\mathcal{F}_{\{n\}}=\left\{t \in[\mathbb{N}]^{<\omega}: n<\min (t) \&\{n\} \cup t \in \mathcal{F}\right\} .
$$

Observe that $\mathcal{B}_{\{n\}}$ is a front on $z / n=\{m \in z: n<m\}$ with rank less than $\alpha$. Consider the function

$$
\Gamma: 2^{\overline{\mathcal{B}}} \times \mathcal{I}^{+} \uparrow z \rightarrow \prod_{n \in \mathbb{N}}\left(2^{\overline{\mathcal{B}_{\{n\}}}} \times \mathcal{I}^{+} \uparrow(z / n)\right)
$$


where $\Gamma(\mathcal{F}, x)=\left(\left(\mathcal{F}_{\{n\}}, x / n\right)\right)_{n \in \mathbb{N}}$ for $x \subseteq z$ in $\mathcal{I}^{+}$. Then $\Gamma$ is Borel. By the inductive hypothesis there is Borel function

$$
S: \prod_{n \in \mathbb{N}}\left(2^{\overline{\mathcal{B}_{\{n\}}}} \times \mathcal{I}^{+} \uparrow(z / n)\right) \rightarrow \prod_{n \in \mathbb{N}}\left(I^{+} \uparrow(z / n)\right)
$$

that satisfies the conclusion of the theorem for each coordinate. Denote by $S_{n}$ the composition of $\Gamma, S$ and the projection to $n$-th coordinate.

We define a sequence of Borel functions $\left\{H_{n}\right\}_{n<\omega}$. For $(\mathcal{F}, x) \in 2^{\overline{\mathcal{B}}} \times \mathcal{I}^{+} \uparrow z$ define recursively

- $H_{0}(\mathcal{F}, x)=S_{0}\left(\mathcal{F}_{\{0\}}, x / 0\right)$,

- $H_{n+1}(\mathcal{F}, x)=S_{n+1}\left(\mathcal{F}_{\{n+1\}}, H_{n}(\mathcal{F}, x) /(n+1)\right)$.

Observe that the map $(\mathcal{F}, x) \mapsto\left\{H_{n}(\mathcal{F}, x)\right\}_{n<\omega}$ is Borel. Since $\mathcal{I}$ is uniformly selective, we can extract, in a Borel way, from the sequence $\left\{H_{n}(\mathcal{F}, x)\right\}_{n<\omega}$ a set $y \in \mathcal{I}^{+}$such that

$$
y / n \subseteq H_{n}(\mathcal{F}, x) \text { for all } n \in y .
$$

Lemma 3.6 naturally provides the notion of $i$-homogeneous for $\mathcal{F}$ for $i=0,1$. Let

$$
y_{i}=\left\{n \in y: H_{n}(\mathcal{F}, x) \text { is } i \text {-homogeneous for } \mathcal{F}_{\{n\}}\right\} .
$$

Then $y_{i}$ is $i$-homogeneous for $\mathcal{F}$. In fact, for $i=0$, let $t$ be a finite subset of $y_{0}$ and let $n=\min (t)$. Then $t / n \subseteq H_{n}(\mathcal{F}, x)$ as $n \in t \subseteq y$. Therefore $t / n \notin \mathcal{F}_{\{n\}}$, as $H_{n}(\mathcal{F}, x)$ is 0 -homogeneous for $\mathcal{F}_{\{n\}}$. Thus $t=\{n\} \cup t / n \notin \mathcal{F}$. Using Lemma 3.6, a similar argument works for $i=1$.

By Lemma 3.6, being $i$-homogeneous for $\mathcal{F}$ is a Borel property, therefore the function $y \mapsto\left(y_{0}, y_{1}\right)$ is Borel. Since $y \in \mathcal{I}^{+}$, then at least one of the sets $y_{0}$ or $y_{1}$ belongs to $\mathcal{I}^{+}$. Let $S(\mathcal{F}, x)=y_{0}$ if $y_{0} \in \mathcal{I}^{+}$and $y_{1}$, otherwise. As $\mathcal{I}^{+}$is Borel, we can pick in a Borel way the alternative that holds. Thus $S$ is Borel.

Since Fin is uniformly selective (Corollary 3.3), we get the uniform version of Nash-Williams' theorem.

Corollary 3.8. Let $\mathcal{B}$ be a front on $\mathbb{N}$. There is a Borel map $S: 2^{\mathcal{B}} \times[\mathbb{N}]^{\omega} \rightarrow[\mathbb{N}]^{\omega}$ such that $S(\mathcal{F}, x)$ is a $\mathcal{F}$-homogeneous subset of $x$, for all $x \in[\mathbb{N}]^{<\omega}$ and all $\mathcal{F} \subseteq \mathcal{B}$.

Using the front $[\mathbb{N}]^{n}$, we get that the classical Ramsey's theorem holds uniformly (the case $n=2$ appeared in [7]).

COROLlary 3.9. For each $n \in \mathbb{N}$, there is a Borel function $S: 2^{[\mathbb{N}]^{n}} \times[\mathbb{N}]^{<\omega} \rightarrow$ $[\mathbb{N}]^{<\omega}$ such that $S(\mathcal{F}, x)$ is an infinite subset of $x$ homogeneous for $\mathcal{F} \subseteq[\mathbb{N}]^{n}$.

Let $\mathcal{C}_{1}$ and $\mathcal{C}_{2}$ be two tall hereditary families with Borel selector. It is easy to verify that $\mathcal{C}_{1} \cap \mathcal{C}_{2}$ has a Borel selector and thus it is natural to ask the following.

QuEsTION 3.10. Let $\mathcal{B}_{1}$ and $\mathcal{B}_{2}$ two fronts on $\mathbb{N}$ and $\mathcal{F}_{i} \subseteq \mathcal{B}_{i}, i \in 2$. Is there a front $\mathcal{B}_{3}$ and $\mathcal{F}_{3} \subseteq \mathcal{B}_{3}$ such that hom $\left(\mathcal{F}_{3}\right) \subseteq \operatorname{hom}\left(\mathcal{F}_{1}\right) \cap \operatorname{hom}\left(\mathcal{F}_{2}\right)$ ?

3.3. Some examples. We present some examples showing that the search for a Borel selector for a tall family $\mathcal{C}$ can be reduced, in some instances, to finding an appropriated coloring $c$ such that $\operatorname{hom}(c) \subseteq \mathcal{C}$ and then use Corollary 3.9.

Let us start recalling that an ideal $\mathcal{I}$ is Katétov below an ideal $\mathcal{J}$, denoted $\mathcal{I} \leq_{K} \mathcal{J}$, if there is a function $f: \mathbb{N} \rightarrow \mathbb{N}$ such that $f^{-1}[x] \in \mathcal{J}$ for every $x \in \mathcal{I}$. This preorder has been extensively investigated (see, for instance, [6] and the references 
therein). Let $\mathcal{R}$ be the ideal on $\mathbb{N}$ generated by the homogeneous sets of the random graph ([7]). It follows from the universal property of the random graph that $\mathcal{R} \leq_{K} \mathcal{I}$ if and only if there is a $\mathcal{F} \subseteq[\mathbb{N}]^{2}$ such that $\operatorname{hom}(\mathcal{F}) \subseteq \mathcal{I}$. In particular, if $\mathcal{R} \leq_{K} \mathcal{I}$, then $\mathcal{I}$ has a Borel selector. All ideals studied in [6,7] are Katětov above $\mathcal{R}$, and therefore they admit a Borel selector. Even Solecki's ideal $\mathcal{S}$ ([13]) has a Borel selector [5] (even thought, it is open whether $\mathcal{R} \leq_{K} \mathcal{S}$ ). It is proved in [5] that having a Borel selector is closed upwards in the Katětov order and if $\mathcal{I}$ is a tall Borel ideal with a Borel selector then there is a tall Borel ideal $\mathcal{J}$ such that $\mathcal{I} \mathbb{Z}_{K} \mathcal{J}$.

ExAmPle 3.11. Let $W O(\mathbb{Q})$ be the collection of all well-ordered subsets of $\mathbb{Q}$ with respect to the usual order. Let $W O(\mathbb{Q})^{*}$ the collection of well ordered subsets of $\left(\mathbb{Q},<^{*}\right)$ where $<^{*}$ is the reversed order of the usual order of $\mathbb{Q}$. Let $\mathcal{C}=W O(\mathbb{Q}) \cup$ $W O(\mathbb{Q})^{*}$. Notice that $\mathcal{C}$ is a complete co-analytic set. To see that $\mathcal{C}$ has a Borel selector, fix an enumeration $\left(r_{n}\right)_{n}$ of $\mathbb{Q}$. Let $c:[\mathbb{Q}]^{2} \rightarrow 2$ be the Sierpinski's coloring which is given by $c\left\{r_{n}, r_{m}\right\}=0$ if and only if $n<m$ and $r_{n}<r_{m}$. Then $\operatorname{hom}(c) \subseteq \mathcal{C}$.

EXAMPLE 3.12. Let $\left(x_{n}\right)_{n}$ be a sequence on a compact metric space $X$. Let

$$
\mathcal{C}\left(x_{n}\right)_{n}=\left\{y \subseteq \mathbb{N}:\left(x_{n}\right)_{n \in y} \text { is convergent }\right\} .
$$

Then $\mathcal{C}\left(x_{n}\right)_{n}$ is clearly tall. We show that there is a coloring $c$ such that hom $(c) \subseteq$ $\mathcal{C}\left(x_{n}\right)_{n}$. In fact, let $f: 2^{\mathbb{N}} \rightarrow X$ be a continuous surjection. Pick $y_{n} \in 2^{\mathbb{N}}$ such that $f\left(y_{n}\right)=x_{n}$ for each $n \in \mathbb{N}$. Let $\preceq$ be the usual lexicografic order on $2^{\mathbb{N}}$. Consider the Sierpinsky coloring $c\{n, m\}_{<}=0$ if and only if $y_{n} \prec y_{m}$. Then $\operatorname{hom}(c) \subseteq \mathcal{C}\left(x_{n}\right)_{n}$.

ExAmple 3.13. Let $(X, \tau)$ be a regular space without isolated points over a countable set $X$. We show that there is a coloring $c:[X]^{2} \rightarrow 2$ such that $\operatorname{hom}(c) \subseteq n w d(X, \tau)$. For $X$ equal to the rationals, the Sierpinski coloring $c$ on $[\mathbb{Q}]^{2}$ satisfies that $\operatorname{hom}(c) \subseteq n w d(\mathbb{Q})$. Now let $X$ be any countable regular space without isolated points. Let $\left(V_{n}\right)_{n}$ be a countable collection of $\tau$-open sets that separates points. Let $\rho$ be the topology generated by the $V_{n}$ 's. Then $(X, \rho)$ is homeomorphic to $\mathbb{Q}$. Therefore the Sierpinski coloring on $\mathbb{Q}$ can be defined on $[X]^{2}$ such that every $c$-homogeneous set is a $\rho$-discrete subset of $X$. Since $\rho \subseteq \tau$, then $\operatorname{hom}(c) \subseteq \operatorname{nwd}(X, \tau)$.

EXAmPle 3.14. Let $e:[\mathbb{N}]^{\omega} \rightarrow \mathbb{N}^{\mathbb{N}}$ be the increasing enumeration function, i.e., $e(x)(n)$ is the nth element of $x$ in its natural order. Notice that $e$ is continuous. Let $\gamma:[\mathbb{N}]^{\omega} \times[\mathbb{N}]^{\omega} \rightarrow[\mathbb{N}]^{\omega}$ be given by

$$
\gamma(x, y)=\{e(x)(n): n \in y\} .
$$

Then $\gamma(x, y) \subseteq x$ and $\gamma$ is continuous. For each $y \in[\mathbb{N}]^{\omega}$, let

$$
\mathcal{C}_{y}=\left\{\gamma(x, y): x \in[\mathbb{N}]^{\omega}\right\} \text {. }
$$

Then $\mathcal{C}_{y}$ is a tall family and obviously $S(x)=\gamma(x, y)$ is a Borel selector for $\mathcal{C}_{y}$.

We will show that $\mathcal{C}_{y}$ contains $\operatorname{hom}(c)$ for some coloring $c$. Let $\left(y_{n}\right)_{n}$ be the increasing enumeration of $y$. We assume that $y_{0} \geq 1$. If $\left(z_{n}\right)_{n}$ is the increasing enumeration of an infinite set $z$, then

$$
z \in \mathcal{C}_{y} \Leftrightarrow(\forall n)\left(y_{n+1}-y_{n} \leq z_{n+1}-z_{n}\right) \& y_{0} \leq z_{0}
$$


Consider the following coloring:

$$
c\{k, l\}=0 \text { if and only if } l-k \geq y_{k} \& k \geq y_{0} .
$$

It is easy to verify that any $c$-homogeneous infinite set is necessarily 0 -homogeneous and also that $\operatorname{hom}(c) \subseteq \mathcal{C}_{y}$.

Let $\mathcal{S}$ be Solecki's ideal ([13]). As we mentioned before, an important open question stated in [6] is whether $\mathcal{R} \leq_{K} \mathcal{S}$. An analogous question is the following.

QUESTION 3.15. Are there a front $\mathcal{B}$ and $\mathcal{F} \subseteq \mathcal{B}$ such that hom $(\mathcal{F}) \subseteq \mathcal{S}$ ?

§4. Negative results. In this section we show that there is a tall $F_{\sigma}$ ideal without a Borel selector and deduce from this fact that there is no uniform version of Galvin's theorem. We also show that there is a $\Pi_{2}^{1}$ tall ideal $\mathcal{I}$ such that $\operatorname{hom}(\mathcal{F}) \nsubseteq \mathcal{I}$ for every $\mathcal{F} \subseteq[\mathbb{N}]^{<\omega}$.

4.1. An $F_{\sigma}$ ideal without a selector and no uniform version of Galvin's theorem. Recall that the hyperspace $K\left(2^{\mathbb{N}}\right)$ serves as a space of codes for $F_{\sigma}$ ideals (see Proposition 2.3). We have seen that the set $\mathcal{T}$ of codes of tall $F_{\sigma}$ ideals is $\Pi_{2}^{1}-$ complete (see Theorem 2.4). To show that there is an $F_{\sigma}$ ideal without a selector we prove that the complexity of the set of codes of $F_{\sigma}$ ideals with a Borel selector is $\Sigma_{2}^{1}$.

We start by modifying a bit the notion of tallness and Borel selector. For $K \in$ $K\left(2^{\mathbb{N}}\right)$, let

$$
\begin{gathered}
\downarrow K=\{x: \exists y \in K x \subseteq y\}, \\
\langle K\rangle^{n}=\left\{\bigcup_{i<n} y_{i}: y_{i} \in K\right\} .
\end{gathered}
$$

Definition 4.1. We say that $K \in K\left(2^{\mathbb{N}}\right)$ is pseudo-tall if for every infinite $x \in 2^{\mathbb{N}}$ there is infinite $y \in \downarrow K$ such that $y \subseteq x$.

One can verify that, as a function, $\downarrow: K\left(2^{\mathbb{N}}\right) \rightarrow K\left(2^{\mathbb{N}}\right)$ is continuous. Note that $K$ is pseudo-tall if and only if $\mathcal{I}_{K}$ is tall.

Lemma $4.2([5])$. Let $K \in K\left(2^{\mathbb{N}}\right)$ with $\emptyset \in K$. There is a Borel function $\phi: 2^{\mathbb{N}} \rightarrow$ $\left(2^{\mathbb{N}}\right)^{\mathbb{N}}$ such that $\phi(x)(n) \in K$ for every $x \in 2^{\mathbb{N}}$ and $n \in \mathbb{N},\{n: \phi(x)(n) \neq \emptyset\}$ is finite for every $x \in 2^{\mathbb{N}}$ and $x \subseteq \bigcup_{n \in \mathbb{N}} \phi(x)(n)$ for every $x \in \mathcal{I}_{K}$.

Proof. For each $n \in \mathbb{N}$, consider the following relation:

$$
R_{n}=\left\{\left(x, y_{1}, \ldots, y_{n}\right) \in 2^{\mathbb{N}} \times K^{n}: x \subseteq y_{1} \cup \cdots \cup y_{n}\right\} .
$$

Notice $R_{n}$ is closed and therefore for each $x \in 2^{\mathbb{N}},\left(R_{n}\right)_{x}=\left\{\left(y_{1}, \ldots, y_{n}\right) \in K^{n}\right.$ : $\left.x \subseteq y_{1} \cup \cdots \cup y_{n}\right\}$ is closed (hence compact). Thus, by the classical theorem of Arsenin-Kunugui (see for instance [8, 35.46]), $R_{n}$ can be uniformized by a Borel function $f_{n}$. That is, letting $P_{n}$ to be the collection of all $x \in 2^{\mathbb{N}}$ such that there is $\left(y_{1} \ldots, y_{n}\right)$ such that $\left(x, y_{1}, \ldots, y_{n}\right) \in R_{n}$, then $P_{n}$ is closed, $f_{n}: P_{n} \rightarrow K^{n}$ is Borel and $\left(x, f_{n}(x)\right) \in R_{n}$ for all $x \in P_{n}$. Notice that $\mathcal{I}_{K}=\bigcup_{n} P_{n}$. Define $\phi(x)$ as follows: if $x \notin \mathcal{I}_{K}$ then $\phi(x)(n)=\emptyset$ for all $n$. On the other hand, if $x \in \mathcal{I}_{K}$, let $\phi(x)=\left(f_{n}(x), \emptyset, \emptyset, \ldots,\right)$ where $n$ is the least $m$ such that $x \in P_{m}$. Then $\phi$ is Borel and satisfies the conclusion.

Let us note, that the previous result is equivalent to saying that there is a Borel function $\mathcal{I}_{K} \rightarrow K^{<\omega}$ such that $x \subseteq \bigcup \varphi(x)(n)$. 
Proposition 4.3. Let $K \in K\left(2^{\mathbb{N}}\right)$ be pseudo-tall. Then $\mathcal{I}_{K}$ has a Borel selector $S$ if and only if it has a Borel selector $S^{\prime}$ such that rng $\left(S^{\prime}\right) \subseteq \downarrow K$.

Proof. Only one implication is not trivial. Let $S$ be a Borel selector for $\mathcal{I}_{K}$. Clearly we can assume without loss of generality that $\emptyset \in K$. Let $\phi$ be the map given by Lemma 4.2. We define a map $W: 2^{\mathbb{N}} \rightarrow \downarrow K$ as follows. For each $x \in \mathcal{I}_{K}$ infinite, let $b_{1}, b_{2}, \ldots, b_{n} \in K$ such that $\phi(x)=\left(b_{1}, \ldots, b_{n}, \emptyset, \ldots\right)$, then we put $W(x)=x \cap b_{i}$ where $i$ is the first $j$ such that $x \cap b_{j}$ is infinite. And $W(x)=\emptyset$, for any other $x \in 2^{\mathbb{N}}$. Then $W$ is a Borel map, $W(x) \subseteq x$ and $W(x)$ is infinite for every infinite set $x \in \mathcal{I}_{K}$. To finish the proof, put $S^{\prime}=W \circ S$.

This leads to a modified definition of a selector.

Definition 4.4. Let $K \in K\left(2^{\mathbb{N}}\right)$ be pseudo-tall. We say that $K$ has a Borel pseudo-selector if there is a Borel function $S: 2^{\mathbb{N}} \rightarrow 2^{\mathbb{N}}$ such that

- $S(x) \in \downarrow K$,

- if $x$ is infinite, then $S(x)$ is also infinite,

- $S(x) \subseteq x$.

By the Proposition 4.3, $K \in K\left(2^{\mathbb{N}}\right)$ has a pseudo-selector if and only if $\mathcal{I}_{K}$ has a selector and therefore it suffices to consider only pseudo-selectors of closed subsets of $2^{\mathbb{N}}$. In other words, the questions of the existence of a Borel selector for $F_{\sigma}$ ideals and for a hereditary tall closed subsets of $2^{\mathbb{N}}$ are equivalent. Let us summarize this in the following proposition.

Proposition 4.5. Let $K \in K\left(2^{\mathbb{N}}\right)$ be tall. The following are equivalent:

- there is a Borel selector for $K$,

- there is a Borel pseudo-selector for $K$,

- the $F_{\sigma}$ ideal $\mathcal{I}_{K}$ has a Borel selector,

- the smallest ideal I that contains $K$ and $\mathbf{F i n}$ has a Borel selector.

Proof. It can be easily verified that the ideal $\mathcal{I}$ in the fourth condition is also $F_{\sigma}$. The only implication that is not clear from the previous argument is how to get a Borel selector from a Borel pseudo-selector. Let $S: 2^{\mathbb{N}} \rightarrow \mathbb{N}$ be a Borel pseudo-selector for $K$. Define

$$
\{(x, y): S(x) \subseteq y \subseteq x, y \in K\} \subseteq 2^{\mathbb{N}} \times 2^{\mathbb{N}} .
$$

This is a Borel set with each vertical section compact and therefore it has a Borel uniformization by a classical uniformization theorem (see, for instance, [8, Theorem 35.46]). The uniformizing function is a Borel selector for $K$.

4.1.1. Coding of Borel functions. Now we are going to present how to code Borel functions. For that end, first we need to code Borel sets. This coding is somewhat standard (see for instance [4, pag. 19]), but we need to present it with some detail. We define a set of labeled well-founded trees which will be the codes of Borel sets.

Definition 4.6. Let $\mathcal{L} T$ be the set of all trees on $\mathbb{N}$ where each node is labeled by an element of $\{0,1\}$.

So, formally, every element of $\mathcal{L} T$ is a tuple $(T, f)$ where $T \subseteq \mathbb{N}<\omega$ is a tree and $f: T \rightarrow 2$. However, we will always write only $T \in \mathcal{L} T$ and $(s, i) \in T$ meaning that $f(s)=i$. 
One can easily check that there $\mathcal{L} T$ is a closed subset of the Polish space of all trees on $\mathbb{N} \times 2$, thus $\mathcal{L} T$ is a Polish space. Moreover, the set of all well-founded labeled trees $W F \mathcal{L} T$ is $\Pi_{1}^{1}$.

We are interested in a closed subspace of $\mathcal{L} T$ which will contain all codes for Borel subsets of $2^{\mathbb{N}}$.

Definition 4.7. Let $\mathcal{L} T_{c} \subseteq \mathcal{L} T$ be the set of all labeled trees satisfying the following condition.

- if $(s, 1) \in T$ then $(s \frown(0), 0) \in T$ and it is the only immediate successor of $(s, 1)$.

One can easily verify that $\mathcal{L} T_{c}$ is a closed subspace of $\mathcal{L} T$ and the set of wellfounded trees $W F \mathcal{L} T_{c} \subseteq \mathcal{L} T_{c}$ is $\Pi_{1}^{1}$.

Now we will define, for each $T \in W F \mathcal{L} T_{c}$, the Borel set $A_{T}$ coded by $T$. And conversely, for each Borel set $A \subseteq 2^{\mathbb{N}}$ there will be a $T \in W F \mathcal{L} T_{c}$ such that $A=A_{T}$. The definition of $A_{T}$ is by recursion on the rank of $T$.

Let $\left\{t_{n}: n \in \mathbb{N}\right\}$ be an enumeration of all basic open sets of $2^{\mathbb{N}}$, i.e., each $t_{n}$ is a finite binary sequence. Recursively define what each $(s, i) \in T$ codes:

- if $(s, 0)$ is a leaf then it codes the basic open set $t_{s(|s|-1)}$ (in the case of $s=\emptyset$, we put $\left.t_{\emptyset(|\emptyset|-1)}=t_{0}\right)$,

- if $(s, 0)$ is not a leaf, then it codes the union of the sets coded by $(s \frown n, i)$ where $\left(s^{\frown} n, i\right) \in T$,

- $(s, 1)$ codes the complement of what $(s \frown(0), 0)$ codes.

Finally, $A_{T}$ is the set coded by $(\emptyset, i)$.

Lemma 4.8. For every Borel set $A \subseteq 2^{\mathbb{N}}$ there is $T \in W F \mathcal{L} T_{c}$ such that $A=A_{T}$. And conversely, $A_{T}$ is Borel for each $T \in W F \mathcal{L} T_{c}$.

Proof. Given $T \in W F \mathcal{L} T_{c}$, one easily shows for induction on the rank of $T$ that $A_{T}$ is Borel. Conversely, given a Borel set $A \subseteq 2^{\mathbb{N}}$, by induction on the Borel complexity of $A$ it is easy to construct a $T \in W F \mathcal{L} T_{c}$ such that $A=A_{T}$.

Let $\mathcal{C}_{i} \subseteq 2^{\mathbb{N}} \times \mathcal{L} T_{c}, i \in 2$, be given by

$$
(x, T) \in \mathcal{C}_{1} \text { if and only if } T \in W F \mathcal{L} T_{c} \text { and } x \in A_{T}
$$

and

$$
(x, T) \in \mathcal{C}_{0} \text { if and only if } T \in W F \mathcal{L} T_{c} \text { and } x \notin A_{T} .
$$

The following is a crucial result.

Lemma 4.9. The relation $\mathcal{C}_{i}$ is $\Pi_{1}^{1}$ for $i \in 2$.

For the proof we need some auxiliary results. We define the following subset $G \subseteq 2^{\mathbb{N}} \times \mathcal{L} T_{c} \times \mathcal{L} T$.

Definition 4.10. A triple $(x, T, S)$ is in $G \subseteq 2^{\mathbb{N}} \times \mathcal{L} T_{c} \times \mathcal{L} T$ if and only if

- $(s, i) \in T$ for some $i \in 2$ if and only if $(s, j) \in S$ for some $j \in 2$,

- if $(s, 0) \in T$ is leaf then $(s, 1) \in S$ if and only if $t_{s(|s|-1)} \sqsubseteq x$,

- if $(s, 1) \in T$ then $(s, 1) \in S$ if and only if $(s \frown(0), 0) \in S$,

- if $(s, 0) \in T$ not a leaf then $(s, 1) \in S$ if and only if there is $n \in \mathbb{N}$ such that $(s \frown(n), 1) \in S$. 
Note that if $(x, T, S) \in G$ then $S$ has the same tree structure as $T$, it only has different labeling. Also note that if $T$ is well-founded then the labeling of $S$ is uniquely determined by the values on its leafs (this can be proved by induction on the rank of $S)$. Since the label of the leafs of $S$ are uniquely determined by $(x, T)$, we can conclude that for each $T \in W F \mathcal{L} T_{c}$ and every $x \in 2^{\mathbb{N}}$ there is exactly one $S$ such that $(x, T, S) \in G$.

Claim 4.11. The set $G$ is Borel.

Proof. We verify that each condition is Borel. The first and the third conditions are independent of the first coordinate and are closed.

For the second condition. Let $P_{s}:=\left\{T \in \mathcal{L} T_{c}: s\right.$ is a leaf of $\left.T\right\}$ and $Q_{s}:=$ $\{T \in \mathcal{L} T:(s, 1) \in T\}$ for each $s \in \mathbb{N}^{<\omega}$. Then $P_{s}$ and $Q_{s}$ are easily seen to be closed. Define

$R_{s}:=\left(2^{\mathbb{N}} \times\left(\mathcal{L} T_{c} \backslash P_{s}\right) \times \mathcal{L} T\right) \cup\left(t_{s(|s|-1)} \times P_{s} \times Q_{s}\right) \cup\left(\left(2^{\mathbb{N}} \backslash t_{s(|s|-1)}\right) \times P_{s} \times\left(\mathcal{L} T \backslash Q_{s}\right)\right)$.

Then $\bigcap_{s \in \mathbb{N}<\omega} R_{s}$ is the collection of all $(x, T, S)$ satisfying the second condition. that

The fourth condition is also independent of the first coordinate and one can verify

$$
Q_{s}^{\prime}:=\left\{T \in \mathcal{L} T:(s, 1) \in T \Longleftrightarrow \exists n \in \mathbb{N}\left(s^{\frown}(n), 1\right) \in S\right\}
$$

is Borel. Combination of $P_{s}, Q_{s}^{\prime}$ and their complements gives us the desired result.

For each $(s, i) \in T$, let $T_{(s, i)}:=\{(t, j):(s \frown t, j) \in T\}$. Consider the following continuous bijection $\Gamma: \mathcal{L} T_{c} \rightarrow \mathcal{L} T_{c}$ where

- if $(\emptyset, 0) \in T$ then $\Gamma(T)=R$ where $(\emptyset, 1) \in R$ and $T_{(\emptyset, 0)}=R_{((0), 0)}$,

- if $(\emptyset, 1) \in T$ then $\Gamma(T)=R$ where $(\emptyset, 0) \in R$ and $T_{((0), 0)}=R_{(\emptyset, 0)}$.

In other words, $\Gamma \uparrow W F \mathcal{L} T_{c}$ is the bijection switching the codes for a set and its complement.

Claim 4.12. Let $T \in W F \mathcal{L} T_{c}$ and $x \in 2^{\mathbb{N}}$ then $|\{S:(x, T, S) \in G\}|=1$ and for the unique $(x, T, S) \in G$ we have that $(\emptyset, 1) \in S$ if and only if $x$ is in the set coded by $T$. Moreover, let $(x, T, S),\left(x, \Gamma(T), S^{\prime}\right) \in G$, then $(\emptyset, 1)$ is in $S$ or $S^{\prime}$ but not in both of them.

Proof. This follows from the discussion after the Definition 4.10 and the definition of $\Gamma$.

Proof of Lemma 4.9. Let $G_{i}:=\{(x, T, S) \in G:(\emptyset, i) \in S\}$ for $i \in 2$. One can easily see that $G=G_{0} \cup G_{1}$ and both sets are Borel. Let $\operatorname{proj}\left(G_{i}\right):=\{(x, T): \exists S \in$ $\left.\mathcal{L} T(x, T, S) \in G_{i}\right\}$. Then from Claim 4.12 we have

$$
\mathcal{C}_{1}=\left(2^{\mathbb{N}} \times W F \mathcal{L} T_{c}\right) \cap \operatorname{proj}\left(G_{1}\right)
$$

and

$$
\mathcal{C}_{0}=\left(2^{\mathbb{N}} \times W F \mathcal{L} T_{c}\right) \cap \operatorname{proj}\left(G_{0}\right) .
$$

Finally, we show that the set $\left(2^{\mathbb{N}} \times W F \mathcal{L} T_{c}\right) \cap \operatorname{proj}\left(G_{i}\right)$ is $\Pi_{1}^{1}$ for $i<2$. This follows from the classical result that if $A \subseteq X \times Y$ is Borel, then $\{x \in X: \exists ! y \in Y(x, y) \in$ $A\}$ is $\Pi_{1}^{1}$. But we can also give a direct proof as follows. 
The sets $H_{i}:=\left(2^{\mathbb{N}} \times \mathcal{L} T_{c}\right) \backslash \operatorname{proj}\left(G_{i}\right)$ are clearly $\Pi_{1}^{1}$ and so are $M_{i}:=W F \mathcal{L} T_{c} \cap$ $H_{i}$ for $i<2$. But then using the Claim 4.12 we see that $\left(2^{\mathbb{N}} \times W F \mathcal{L} T_{c}\right) \cap \operatorname{proj}\left(G_{i}\right)=$ $M_{1-i}$.

Next we define a coding of Borel functions from $2^{\mathbb{N}}$ to $2^{\mathbb{N}}$. Let

$$
C_{n}:=\left\{x \in 2^{\mathbb{N}}: x(n)=1\right\} .
$$

Let $f: 2^{\mathbb{N}} \rightarrow 2^{\mathbb{N}}$ be a Borel function and let $A_{n}:=f^{-1}\left(C_{n}\right)$. Then $f$ is described by the sequence $\left\{A_{n}\right\}_{n \in \omega}$ because $f(x)(n)=1$ if and only if $x \in A_{n}$. Thus, the following is the natural definition of codes for Borel functions.

DeFINITION 4.13. Let $\mathcal{F} T=\left(\mathcal{L} T_{c}\right)^{\mathbb{N}}$ and $W F \mathcal{F} T=\left(W F \mathcal{L} T_{c}\right)^{\mathbb{N}}$.

The product topology on $\mathcal{F} T$ is Polish and $W F \mathcal{F} T \subseteq \mathcal{F} T$ is $\boldsymbol{\Pi}_{1}^{1}$. We denote the elements of $\mathcal{F} T$ also by $T$ and the $n$-th element of $T$ as $T(n)$.

Lemma 4.14. The set WFFT codes Borel functions from $2^{\mathbb{N}}$ to $2^{\mathbb{N}}$ i.e., every sequence $T \in W F \mathcal{F} T$ is a code for a function $f_{T}$ and for every Borel function $f$ there is a sequence $T \in W F \mathcal{F} T$ such that $f_{T}=f$.

Proof. As it was mentioned above, every Borel function $f$ is coded by a sequence of Borel sets $\left(A_{n}\right)_{n}$. Let $T=(T(n))_{n}$ be such that $T(n) \in W F \mathcal{L} T_{c}$ codes $A_{n}$ for each $n \in \mathbb{N}$.

4.1.2. Coding of selectors and $F_{\sigma}$ ideals. Now we will show that the codes for $F_{\sigma}$ ideals with Borel selector is $\Sigma_{2}^{1}$ and then conclude with the main results of this section.

Consider the following map $\Omega: 2^{\mathbb{N}} \times W F \mathcal{F} T \rightarrow 2^{\mathbb{N}}$ given by $\Omega(x, T)(n)=1$ if and only if $x$ is in the set coded by $T(n)$. From the definitions of $\mathcal{C}_{i}, \Omega$, and Lemma 4.9 the following is straightforward.

Lemma 4.15. Let $\mathcal{R} \subseteq 2^{\mathbb{N}} \times \mathcal{F} T \times 2^{\mathbb{N}}$ be given by $(x, T, y) \in \mathcal{R}$ if and only if

$$
\forall n \in \mathbb{N}\left[\left((x, T(n)) \in \mathcal{C}_{1} \rightarrow y(n)=1\right) \wedge\left((x, T(n)) \in \mathcal{C}_{0} \rightarrow y(n)=0\right)\right] .
$$

Then $\mathcal{R}$ is $\Sigma_{1}^{1}$ and for all $(x, T, y) \in 2^{\mathbb{N}} \times W F \mathcal{F} T \times 2^{\mathbb{N}}$ we have

$$
\Omega(x, T)=y \Longleftrightarrow(x, T, y) \in \mathcal{R} .
$$

Consider the following set $\mathcal{M} \subseteq 2^{\mathbb{N}} \times \mathcal{F} T \times K\left(2^{\mathbb{N}}\right)$ defined by $(x, T, K) \in \mathcal{M}$ if and only if

- $T \in W F \mathcal{F} T$,

- $\Omega(x, T) \in \downarrow K$,

- $\Omega(x, T) \subseteq x$,

- if $|x|=\omega$, then $|\Omega(x, T)|=|x|$.

Lemma 4.16. $\mathcal{M}$ is a $\Pi_{1}^{1}$ subset of $2^{\mathbb{N}} \times \mathcal{F} T \times K\left(2^{\mathbb{N}}\right)$.

Proof. It follows from Lemma 4.15. For instance, the second condition can be expressed as follows:

$T \in W F \mathcal{F} T \wedge \Omega(x, T) \in \downarrow K \Longleftrightarrow T \in W F \mathcal{F} T \wedge \forall y \in 2^{\mathbb{N}}((x, T, y) \in \mathcal{R} \rightarrow y \in \downarrow K) . \quad \dashv$ $\Sigma_{2}^{1}$

THEOREM 4.17. The set of all $K \in K\left(2^{\mathbb{N}}\right)$ that have a Borel pseudo-selector is 
Proof. This set may be described as

$$
\left\{K \in K\left(2^{\mathbb{N}}\right): \exists T \in \mathcal{F} T \forall x \in 2^{\mathbb{N}}(x, T, K) \in \mathcal{M}\right\}
$$

which is $\boldsymbol{\Sigma}_{2}^{1}$.

THEOREM 4.18. There is an $F_{\sigma}$ tall ideal without a Borel selector.

Proof. The codes of $F_{\sigma}$ ideals with a Borel selector are clearly a subset of all tall $F_{\sigma}$ ideals and the former set is $\Sigma_{2}^{1}$ but the latter is $\Pi_{2}^{1}$-complete (see Theorem 2.4).

Corollary 4.19 ([8]). There is a closed subset of $A \subseteq \mathbb{N}^{\mathbb{N}} \times \mathbb{N}^{\mathbb{N}}$ such that $\mathbb{N}^{\mathbb{N}}=$ $\operatorname{proj}(A)=\left\{x \in \mathbb{N}^{\mathbb{N}}: \exists y \in \mathbb{N}^{\mathbb{N}}\right.$ s.t. $\left.(x, y) \in A\right\}$ and it does not have a Borel uniformization .

Proof. The space $X:=2^{\mathbb{N}} \backslash\{x: \exists n$ s.t. $\forall m>n x(m)=0\}$ is homeomorphic to $\mathbb{N}^{\mathbb{N}}$. The restriction of the relation $S=\left\{(x, y) \in 2^{\mathbb{N}} \times 2^{\mathbb{N}}: x \supseteq y\right\}$ to $X$ is closed in $X$. By our theorem there is a tall $K \in K\left(2^{\mathbb{N}}\right)$ without Borel selector. Then $K \cap X$ is closed in $X$ and the closed set $A:=S \uparrow(X \times X) \cap(X \times(K \cap X))$ has no Borel uniformization.

Since Theorem 4.18 has an indirect proof we have the following.

QUESTION 4.20. Find a concrete example of an $F_{\sigma}$ tall ideal without a Borel selector.

4.1.3. Galvin's theorem. Now we use some previous results to simply observe that there is no uniform version of Galvin's theorem.

THEOREM 4.21. There is $\mathcal{F} \subseteq[\mathbb{N}]^{<\omega}$ such that there is no Borel function $S: 2^{\mathbb{N}} \rightarrow$ $2^{\mathbb{N}}$ satisfying $S(x) \in \operatorname{hom}(\mathcal{F}), S(x) \subseteq x$ and $S(x)$ infinite for every infinite $x \in 2^{\mathbb{N}}$.

Proof. Combine Theorem 4.18 and Proposition 2.8.

4.2. $\mathrm{A} \Pi_{2}^{1}$ tall ideal without a closed tall subset. We construct a $\Pi_{2}^{1}$ tall ideal which does not contain $\operatorname{hom}(\mathcal{F})$ for every $\mathcal{F} \subseteq[\mathbb{N}]^{<\omega}$. Recall that $h o m(\mathcal{F})$ is $\Pi_{1}^{1}$ for every $\mathcal{F} \subseteq[\mathbb{N}]^{<\omega}$ and therefore we have the following.

ObSERVATION 4.22. Let $R \subseteq 2^{[\mathbb{N}]^{<\omega}} \times[\mathbb{N}]^{\omega} \times[\mathbb{N}]^{\omega}$ be defined by

$$
R(\mathcal{F}, x, y) \Leftrightarrow y \subseteq x \& y \in \operatorname{hom}(\mathcal{F}) .
$$

Then $R$ is $\mathbf{\Pi}_{1}^{1}$.

LEMmA 4.23. [7, Lemma 4.6] There is a continuous function $\psi:[\mathbb{N}]^{\omega} \times 2^{\mathbb{N}} \rightarrow 2^{\mathbb{N}}$ such that for every infinite $x \in[\mathbb{N}]^{\omega}$, the collection $\left\{\psi(x, y): y \in 2^{\mathbb{N}}\right\}$ is an almost disjoint family of infinite subsets of $x$. Moreover, for all infinite $x$ there is an infinite $z \subseteq x$ such that $z \cap \psi(x, y)=\emptyset$ for all $y \in 2^{\mathbb{N}}$.

THEOREM 4.24. There is $a \Pi_{2}^{1}$ tall ideal $\mathcal{I}$ such that for all $x \in \mathcal{I}^{+}$and all $\mathcal{F} \subseteq[\mathbb{N}]^{<\omega}$ there is $y \subseteq x$ with $y \in \operatorname{hom}(\mathcal{F}) \cap \mathcal{I}^{+}$. In particular, $\mathcal{I}$ does not contain any closed hereditary tall set.

Proof. The construction is similar to that presented in [7, Theorem 4.7]. Let $\varphi: 2^{\mathbb{N}} \rightarrow 2^{[\mathbb{N}]^{<\omega}}$ be a continuous surjection. By the classical uniformization theorem [8], let $R^{*} \subseteq R$ be a $\Pi_{1}^{1}$ uniformization for the relation $R$ given by Observation 4.22. Let $\psi$ be given by Lemma 4.23. Let 


$$
\begin{gathered}
\mathcal{C}_{1}=\left\{y \in[\mathbb{N}]^{\omega}: \exists x \in 2^{\mathbb{N}}, R^{*}(\varphi(x), \psi(\mathbb{N}, x), y)\right\}, \\
\mathcal{C}_{n+1}=\left\{y \in[\mathbb{N}]^{\omega}: \exists x \in 2^{\mathbb{N}}, \exists z \in \mathcal{C}_{n}, R^{*}(\varphi(x), \psi(z, x), y)\right\} .
\end{gathered}
$$

Then each $\mathcal{C}_{n}$ is $\boldsymbol{\Sigma}_{\mathbf{2}}^{\mathbf{1}}$. Finally, let

$$
x \in \mathcal{H} \Leftrightarrow(\exists n \in \mathbb{N})\left(\exists y \in \mathcal{C}_{n}\right) y \subseteq^{*} x .
$$

To see that $\mathcal{I}$ is an ideal, suppose $x \cup y \notin \mathcal{I}$, we will show that either $x \notin \mathcal{I}$ or $y \notin \mathcal{I}$. Let $n \in \mathbb{N}$ and $z \in \mathcal{C}_{n}$ be so that $z \subseteq^{*} x \cup y$. Consider the following coloring of pairs:

$$
c\{n, m\}=1 \text { if and only if }\{n, m\} \subseteq x .
$$

Let $w \in 2^{\mathbb{N}}$ be such that $\varphi(w)=c$, and let $u \in[\mathbb{N}]^{\omega}$ be such that $R^{*}(\varphi(w), \psi(z, w), u)$. Then $u \in \mathcal{C}_{n+1}$ and it is $c$-homogeneous. If $u$ is 1 homogeneous, then $u \subseteq x$ and if $u$ is 0 -homogeneous, then $u \cap x$ has at most one point. Since $u \subseteq z \subseteq^{*} x \cup y$, then $u \subseteq^{*} x$ or $u \subseteq^{*} y$, so either $x \notin \mathcal{I}$ or $y \notin \mathcal{I}$.

Let us see that $\mathcal{I}$ is tall. Fix $x \notin \mathcal{I}$. Then there is $n \in \mathbb{N}$ and $y \in \mathcal{C}_{n}$ such that $y \subseteq{ }^{*} x$. By Lemma 4.23, there is $z \subseteq y$ infinite such that $\psi(y, x) \cap z=\emptyset$ for all $x \in 2^{\mathbb{N}}$. We claim that $z \in \mathcal{I}$. In fact, towards a contradiction, let $m>n$ and $w \in \mathcal{C}_{m}$ such that $w \subseteq \subseteq^{*} z$. As $\mathcal{C}_{m}$ is a.d., then there is $u$ such that $w \subseteq^{*} \psi(y, u)$, which is impossible.

We show that it satisfies the other requirements. It is clearly $\Pi_{2}^{1}$. Let $\mathcal{F} \subseteq[\mathbb{N}]^{<\omega}$ and $y \notin \mathcal{I}$. Then there is $x \in 2^{\mathbb{N}}$ such that $\mathcal{F}=\varphi(x)$. There is also $n \in \mathbb{N}$ and $z \in \mathcal{C}_{n}$ so that $z \subseteq^{*} y$. Let $w$ be such that $R^{*}(\varphi(x), \psi(z, x), w)$. Then $w \subseteq z$ and is $\mathcal{F}$-homogeneous. By definition, $w \in \mathcal{H}$. Then $w \cap y$ is infinite and $\mathcal{F}$-homogeneous.

The last claim follows from Lemma 2.8 .

A corollary of the proof of the previous theorem provides a more general construction of co-analytic tall ideals as in [7].

TheOREM 4.25. Let $\mathcal{B}$ be a front over $\mathbb{N}$. There is a co-analytic tall ideal $\mathcal{I}$ such that hom $(\mathcal{F}) \nsubseteq \mathcal{I}$ for all $\mathcal{F} \subseteq \mathcal{B}$.

Proof. From the proof of Theorem 4.24 and using Corollary 3.8 instead of the co-analytic uniformizing set $R^{*}$, we define the sets $\mathcal{C}_{n}$, which are now analytic. Thus, the ideal constructed is co-analytic.

In [7] it was asked whether every analytic tall ideal contains a $F_{\sigma}$ tall ideal. A weaker version of this question is the following.

Question 4.26. For which tall families $\mathcal{C}$ there is $\mathcal{F} \subseteq[\mathbb{N}]^{<\omega}$ such that hom $(\mathcal{F}) \subseteq \mathcal{C}$ (here $\operatorname{hom}(\mathcal{F})$ is not necessarily closed $)$ ?

Acknowledgments. The first author would like to thank the members of Prague Set theory seminar for many useful suggestions and comments on the preliminary version of this paper. The second author would like to thank Stevo Todorčević for the discussions they had years ago about some of the results presented in this article.

The first-listed author was supported by the GACR project 15-34700L and RVO: 67985840 .

The second author thanks Vicerrectoría de Investigación y Extensión de la Universidad Industrial de Santander for the financial support for this work, which is part of the VIE project \#2422. 


\title{
REFERENCES
}

[1] J. Avigad, An effective proof that open sets are Ramsey. Archive for Mathematical Logic, vol. 37 (1998), no. 4, pp. 235-240.

[2] H. Becker, S. Kahane, and A. Louveau, Some complete $\Sigma_{2}^{1}$ sets in harmonic analysis. Transactions of the American Mathematical Society, vol. 339 (1993), no. 1, pp. 323-336.

[3] F. Galvin and K. Prikry, Borel sets and Ramsey's theorem, this Journal, vol. 38 (1973), pp. 193-198.

[4] S. GaO, Invariant Descriptive Set Theory, Pure and Applied Mathematics, vol. 293, CRC Press, Boca Raton, FL, 2009.

[5] J. Grebík and M. Hrušák, No minimal tall Borel ideal in the Katětov order, 2017, arxiv.org/pdf/1708.05322.pdf.

[6] M. HRušák, Katětov order on Borel ideals. Archive for Mathematical Logic, vol. 56 (2017), no. 7-8, pp. 831-847.

[7] M. Hrušák, D. Meza-Alcántara, E. Thümmel, and C. Uzcátegui, Ramsey type properties of ideals. Annals of Pure and Applied Logic, vol. 168 (2017), no. 11, pp. 2022-2049.

[8] A. S. Kechris, Classical Descriptive Set Theory, Springer-Verlag, New York, 1994.

[9] A. R. D. Mathias, Happy families. Archive for Mathematical Logic, vol. 12 (1977), pp. 11-59.

[10] K. MAZur, $F_{\sigma}$-ideals and $\omega_{1} \omega_{1}^{*}$-gaps in the Boolean algebras $P(\omega) / I$. Fundamenta Mathematicae, vol. 138 (1991), no. 2, pp. 103-111.

[11] C. St. J. A. Nash-Williams, On better-quasi-ordering transfinite sequences. Mathematical Proceedings of the Cambridge Philosophical Society, vol. 64 (1968), no. 2, pp. 273-290.

[12] J. Silver, Every analytic set is Ramsey, this Journal, vol. 35 (1970), pp. 60-64.

[13] S. SOLECKI, Filters and sequences. Fundamenta Mathematicae, vol. 163 (2000), no. 3, pp. 215-228.

[14] R. Solovay, Hyperarithmetically encodable sets. Transactions of the American Mathematical Society, vol. 239 (1978), pp. 99-122.

[15] S. TODORČEvić, Higher dimensional Ramsey theory, Ramsey Methods in Analysis (S. Argyros and S. Todorčević, editors), Advanced Courses in Mathematics, CRM Barcelona, Birkhäuser, Basel, 2005.

[16] - Introduction to Ramsey Spaces. Annals of Mathematical Studies, vol. 174. Princeton University Press, Princeton, NJ, 2010.

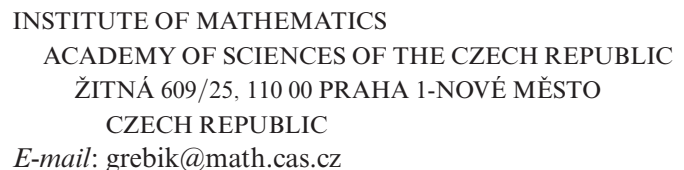

\author{
ESCUELA DE MATEMÁTICAS \\ UNIVERSIDAD INDUSTRIAL DE SANTANDER \\ CRA. 27 CALLE 9 UIS EDIFICIO 45 \\ BUCARAMANGA, COLOMBIA
}

E-mail: cuzcatea@saber.uis.edu.co 